\title{
A Review Study on Bicomponent (Bico) Fibre/ Filament
}

\author{
Md. Khalilur Rahman Khan ${ }^{1 *}$ and Mohammad Naim Hassan ${ }^{2}$ \\ ${ }^{1}$ Department of Textile Engineering, Bangladesh University of Business and Technology, Bangladesh \\ ${ }^{2}$ Department of Textile Engineering, Khulna University of Engineering and Technology, Bangladesh
}

*Corresponding author: Md. Khalilur Rahman Khan, Department of Textile Engineering, Bangladesh University of Business and Technology, Dhaka, Bangladesh.
Received Date: April 01, 2021

Published Date: May 04, 2021

\begin{abstract}
Bi-component fibers are fibers that in a single fiber consist of two distinct raw material components. It is made by simultaneously spinning two compositions in each capillary of the spinneret. Core-sheath (C/S), side-by-side (S/S), segmented-pie (orange) and islands in-the-sea (I/S) are the most common types of bico fibers. The growing demand for non-woven fabrics is one of the growth drivers of the global bi-component fiber industry. In addition, bico fibers are also used in the manufacture of bulky goods, microfiber fabrics, advanced textiles, etc. Various types of commercial bico fibers as well as bico fiber fabrics are manufactured by many companies around the world. However, different aspects of bico fibers, their suitability in various fields of application, and some commercially available bico fibers are briefly reviewed in this paper.
\end{abstract}

Keywords: Bicomponent fiber; Core-sheath fiber; Nonwoven; Microfiber

\section{Introduction}

Fibers have been mixed for several years to produce fabrics that incorporate the properties of both fibers. The cotton/polyester blend common in shirts is a good example. Bicomponent fibers or Bico fibers are typically synthetic. The American Society of Testing Materials (ASTM) recognizes a bicomponent fiber as a fiber composed of two polymers that are chemically or physically distinct (or both) [1]. 'Composite', 'conjugate' or 'hetro' fibers are often referred to as bicomponent fibers [2]. The invention of this fiber structure was influenced by the structure of wool [3]. Biocomponents are used when characteristic, such as dyeability, soil resistance or heat insulating properties, is necessary for the surface, while the core contributes to strength, flexibility or some other required characteristic. Bico fibers of staple, filament \& microfiber forms are available [4]. These fibers have been typically produced for nonwovens, composites and reinforced fiber products. The key growth drivers of the bicomponent fiber market are rising consumer quality of life, growing nonwoven products, the demand for baby and adult diapers, increasing automotive production, increasing awareness of personal care products, increasing demand for softer and bulkier products, and better performance of bicomponent fibers over single polymer fibers [5].

In the textile industry, comprehension is the fuel for creativity and success. From this viewpoint, an overview of the fundamental knowledge of bico fiber and the key areas of application is attempted in this study. Some concise information on commercial bi-component fibers are also mentioned in this paper.

\section{Principle of Bico Fiber Production}

Two distinct polymers are spun into a bicomponent fiber [6]. Bico fibers are generally processed by melt spinning system [1]. These fibers are formed by extruding two polymer components simultaneously and continuously from two different extruders to 
create unitary filament strands from the same spinning orifices of a spinneret. The two components have a separate interface along the fiber axis, instead of being homogeneously blended, and the two sections can have different configurations or patterns [7]. Several factors determine the cross-section of a bicomponent fiber: the polymer distribution/metering spin pack of the plate, the adjusting of the two polymer viscosities, and the interfacial tension between those polymers [1]. The quality and properties of the materials used, their arrangement in the fiber, their relative proportions and the obtained thickness of the fiber decide the characteristics of the bicomponent fibers [8]. The properties of bico fibers can be engineered by introducing new functionality through additives (e.g., chemicals etc.), surface treatment and new or additional processes. This fiber may be applied to the hydrophobic or hydrophilic feature through special manufacturing techniques. The ratios of the polymer viscosities should not, however, be too disparate to guarantee the spinning efficiency. Substantial viscosity discrepancies result in spinning errors viz. 'dog legs' in the case of side-by-side processes that generally lead to the bending of the melt flow just below the spinneret, for example [9].

\section{Types of Bico Fiber}

A wide number of potential cross-sections are provided by bicomponent yarns. Core-sheath, side-by-side, segmented-pie and islands in the sea are the four best known forms of bico fiber (Figure 1). Typical and often used in staple fiber yarns are core-sheath and side-by-side bico fibers [9]. In the global two- component fiber industry, the sheath/core structure holds the highest share. The segmented pie and islands in the sea have more technically advanced bicomponent fiber structures [10]. These two types are mainly produced for the manufacture of super-microfibers, i. e. Less than dpf 0.3 [9]. There are special features and applications for each type of bi-component fiber [7].

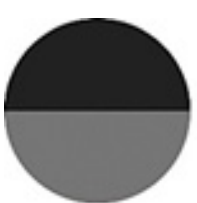

a)

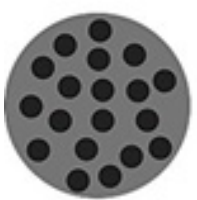

c)

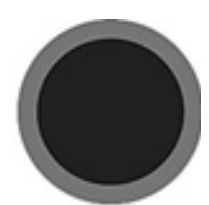

b)

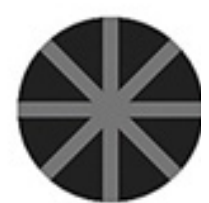

d)

Figure 1: Common types of Bico Fiber $(a=S / S, b=C / S, C=S / I \& d=$ Segmented Pie fiber).

\section{C/S Bico fiber}

The majority of commercially generated bicomponent fibers are currently sheath-core, which assumes various spatial configurations depending on the core position [11]. The core component is completely surrounded by the sheath component in $\mathrm{C} / \mathrm{S}$ bicomponent fibers [12]. In general, the polymers used in the core have the maximum elasticity module values than the material of the sheath, and the core transversal area is greater than the sheath [11]. Depending on the fabric properties required, the structure of the core is either eccentric or concentric. The concentric type is chosen if high fabric strength is needed [12]. If the inner fiber is not centered, but offset to one side, an improved crimp can be developed of the filaments, and even three-dimensional crimps are possible [13]. The ability to produce a surface with the desired luster, dyeability, and handle characteristics while maintaining a core that dominates the tensile properties, is one of the advantages of $\mathrm{C} / \mathrm{S}$ bico fibers [12]. The key factors driving the demand for sheath/core structure in the bicomponent fiber sector are strong interlocking capabilities and less reactive to moisture. In comparison, raw materials are concerned for a very high proportion of the cost of producing fibers and filaments [9]. From that viewpoint, by designing the variable proportions of the two polymer components or using less costly polymers such as B-grade polymer or recycled material, the core sheath structure often provides a way of minimizing the cost [9, 12]. C/S bi-component staple fibers, known in Japan as ESC fibers, are primarily targeted at air through bonding and thermos bonding applications. At an elevated temperature, the sheath of the fibers with a lower melting point than that of the core melts, causing bonding points with nearby fibers, either bi-component or monocomponent [3]. Reducing the sheath ratio is usually desirable. However, most such applications have previously used a sheath ratio of up to 50 percent due to hardware and process limitations of older spinning equipment. A sheath ratio of 10 percent or less can be commercially developed with today's technology. PET/PE, PET/PBT, PET/Co-PET, PLA/Co-PLA, PLA/PBS etc. are the most prominent sheath core combinations. 


\section{S/S Bico fiber}

Side-by-side (S/S) bicomponent fibers are formed by spinning two fiber components together simultaneously, as the names indicate, so that they are bound longitudinally. Both components may differ in their chemical composition, and they may even differ in certain properties, such as molecular weight or degree of crystallization, allowing for differential expansion or shrinkage [7]. S/S bico fibers and filaments are primarily developed in the form of circular or circular-hollow cross sections [9]. Within the filament with a different strain level or shrinkage tendency, selfbulking is generated by two polymers. The two polymers' distinct shrinkage rate causes the fiber, when heated under relaxation, to curl into a helix. S/S bico fibers are highly porous and stretchable [3]. In staple fiber/fill applications, side-by-side self-bulking fibers are now commonly used. In filament applications such as textured upholstery yarns, such items have also recently been made available [14]. Cord fabrics are traditional textile fabrics made from S/S bico yarns, for instance [9]. These fibers may be used to create split-able fibers with distinct cross-sections that generate finer fibers [10].

\section{Orange Bico fibers}

In the segmented-pie method, when subjected to mechanical stress, a bicomponent fiber is spun from two incompatible polymers which adhere poorly then split into microfibers [15]. It is also called orange bico fiber. Most generally, filaments with 16 segments are used. However, it is also possible to produce filaments comprising 32 or 64 segments [9]. Filaments composed of alternating segments of two separate polymers (pies or stripes) breaking apart during downstream processing. For example, mild chemical treatment in dyeing and break-up by hydro-entanglement of staple or spun bond fibers are examples of downstream processes used to split such fibers [14]. For most microfiber goods, segmented pie fibers are the base [10]. Very fine fibers having high strength and high surface areas are possible to produce by this type of bico fiber [10]. Microfiber production through direct spinning is difficult and costly, as throughputs are low. But a pie- wedge fiber allows high throughput rate and is robust during fiber and fabric production.

\section{S/I Bico fiber}

One type of bicomponent fiber is S/I Bico Fiber, whereby several fibrils of one polymer are distributed in another polymer's matrix. Fibrils are referred to as islands and the sea is the matrix [12]. Within the industry, the existing standard is 37 islands per filament, while construction with up to 91 islands is possible at Oerlikon Neumag [9]. The sea component dissolved or melted away in subsequent processing [14]. Toray developed this technique at first [16]. These items have mostly been staple fibers in nonwoven fabrics with the sea dissolved away. Promising potential is also created by the use of hydroentangling to split the islands away from the sea [14]. The solvent is passed into a cloth made of sea / island fibers, and a microfiber fabric is the result. The size of the resulting fibers decreases as the number of islands increases.

\section{Application Areas of Bico Fiber}

Many fiber applications have been revolutionized by Bicomponent technologies. Products have been made lighter, stronger and simpler to work with. This type of fiber is expected to develop as an advanced material across many end-use applications, including hygiene, textiles, automotive, home furnishings, and many others to solve problems and meet customer needs [10]. In nonwovens, bi-component staple fibers have been one of the most significant fibers. In various end-use industries, there has been a rapid advancement in non-woven technology, thereby driving the market for non-woven fabrics that provide more comfort and softness. With continuous innovation in goods to suit market demand, bicomponent fibers have been at the forefront in meeting such requirements. In all applications where there is direct interaction of sensitive human skin with non-woven fabric (e.g. baby nappies, ladies' grooming and incontinence nappies and towels), the advantages of bico nonwoven fabrics are present [17]. Bicomponent filaments in traditional textiles such as suede and chamois, as well as in nonwovens, are used in China. Fibers are normally used as binder fibers in technical textiles to tie various fibers and layers together to create sophisticated textile solutions made from the finest potential fiber blends.

Bicomponent techniques in which the fibers are either caused to break apart or one of the two components is dissolved or melted away may create microfibers [14]. In this case, the polymers must be incompatible, and their adhesiveness should be weak [4]. It has long been recognized that these methods are capable of generating much smaller fibers than with homopolymer techniques. The pie wedge cross sections are usually used for the processing of microfibers. Microfibers are also formed by the sea/islands cross section. With modern technology, it is now possible to manufacture such microfibers at costs comparable to traditional microfibers [14].

Due to increased specific surface area, increased surface adsorption power, and improved filtration performance, finer fibers in many applications are increasingly desirable in the nonwoven industry. One of the popular subjects in nonwoven industries has been the splitting of bicomponent (Bico) fibers to obtain finer fibrous non-woven webs [7]. They have even more properties when microfiber is made into nonwovens: strong isolation, impermeability, breathing capability, wrinkle-resistance, stain-resistance, quick ability to wash. Their considerably higher comfort and versatility offer many field applications [18].

The additional application of bicomponent fibers consists of improving the structure of disposable hygienic absorbent products, such as disposable diapers, particularly in a liquid permeating layer. As the absorbent layer is typically made from an absorbent material that includes natural fibers such as cellulose, fluff pulp 
fibers, cotton fibers, and rayon fibers, and a super absorbent polymer (SAP), the absorbent material of the absorbent layer tends to expand. As a result, it become heavy causing an uncomfortable feeling in user as liquid is absorbed into the absorbent layer. So, the synthetic fibers are inserted into the absorbent layer in order to introduce a supportive structure to fix the absorbent material in place [11].

The bicomponent fiber is among the few man-made fibers that report a relatively low impact of the pandemic on their demand, considering the widespread impact of the pandemic. Growing knowledge of health and hygiene among people around the world is fueling the growth in demand for different hygiene products. Bicomponent fiber effectively fulfills main requirements for hygiene products, such as softness, improved fluid absorption, low skin discomfort, high strength, and high bulkiness [19]. Three staple fiber bicomponent systems have been successfully commissioned in China by Oerlikon Neumag. The systems are used to produce core-sheath bi-component fibers, with capacities of 50 tons per day each [20].

\section{Commercial Bico Fiber}

Bicomponent fibers are primarily sold as bonding components in thermo-bonded nonwoven fabrics, as self- crimping fibers to achieve textured yarn, or as fibers with the surface functionality of special polymers and additives at a reduced cost, depending on the characteristics of the different polymers [15]. The first commercial bi-component applications were introduced by DuPont. This was a "Cantrese" side-by-side hosiery yarn and was made of two nylon polymers, which formed a strongly coiled elastic fiber upon retraction. However, the first commercial use of bico fiber, 'Heterofil' (ICI) was in carpets and upholstery fabrics. It was the sheath-core binding fiber consisting of a lower melting temperature sheath (Nylon 6, $220^{\circ} \mathrm{C}$ ) and a higher melting temperature core (Nylon 66, $260^{\circ} \mathrm{C}$ ) [3]. ES fiber, also called PP/PE composite fiber, is the most widely available bicomponent fiber, which can be used by distinct heat treatment for various non- woven fabrics. ES FiberVision, Huvis Corporation, Far Eastern New Century, Jiangnan High Polymer Fiber Co., Ltd., CHA Technologies Group and Jiaxing Xinwei Chemical Fiber Co., Ltd., Freudenberg Group, Toray Industries, Inc., Indorama Ventures Public Company Limited, Swicofil, EMS-CHEMIE HOLDING AG, Eastman, DuPont, Fiber Innovation Technology Hyosung etc. are the major bico fiber manufacturers.

\section{Lycra T400®}

Innovative bicomponent polyester filaments sold as Lycra T400® by the DuPont Company have recently gained significant attention. This S/S bico filaments yarn is composed of $60 \%$ polyethylene terephthalate (PET) and $40 \%$ polytrimethylene terephthalate (PTT) [8]. In applications that require low to moderate stretch, LYCRA ${ }^{\circledR}$ T $400 \AA$ fiber offers outstanding efficiency and aesthetics. Each polymer shrinks to a different degree when exposed to heat, creating a smooth helical crimp. The stretch and recovery of LYCRA $®$ T $400 \AA$ fiber is greater and more robust than those of textured yarns since the crimp is not mechanically generated. The regularity of the crimp allows the improved fabric aesthetics. LYCRA ${ }^{\circledR}$ T $400 \AA$ fiber is highly resistant to chemicals, including chlorine, and UV radiation. Dyeing characteristics resemble to that of textured polyester. LYCRA ${ }^{\circ}$ T400® fiber's unique structure also offers the added advantage of good moisture control to make wearers stay cool and dry. It is applied for denims, job wear, shirting's, circular knits, and socks and other lightweight woven [21].

\section{ETC-bounce -" Air-trapping" bico fiber}

ES FIBERVISIONS developed ETC-Bounce fiber - polyethylene/ polyester bicomponent fiber. Compared to polyolefin polymers, polyester (PET) has a much higher melting point and resiliency. Consequently, after an applied load is released, the fibers have a' bounce back effect'. High bulkiness and resilience give the nonwoven excellent liquid acquisition efficiency by optimum achieved fiber properties. This difference in the melting point between the sheath and the core provides the fabric with high heat-sealing efficiency, as its polyester core remains intact even after the heat-sealing process and gives the sealed component high tensile strength. Compared to other fabrics, the ETC-Bounce achieves greater bulkiness and resilience [22].

\section{PTC bicomponent fiber}

The integration of polyester and polypropylene in the PTC bicomponent fiber from ES FIBERVISIONS provides outstanding bulkiness, resilience, ultrasonic bond ability and processability of a wide range of conditions. In a range of applications, such as hygiene acquisition-distribution layers, filters, household goods, medical products and other through-air (oven) bonded products, as well as in carded thermal bonded products, PTC fiber can be used successfully [22].

\section{Soft bico}

The Soft Bico from ES FIBERVISIONS is a mainly polypropylene/ polyethylene bi-component fiber that provides built-in softness, robust hydrophilicity and wettability. The melting point difference between the sheath and core polymers $\left(130{ }^{\circ} \mathrm{C}\right.$ for polyethylene and $160{ }^{\circ} \mathrm{C}$ for the polypropylene core) allows for good bonding of the sheath while the core remains intact [22].

\section{CM800}

Part of CHA Technologies, Haining Xin Gao Fibers, China, is the manufacturer of specialty bico stretch filament yarns, i.e. CM800, a new form of stretch yarn made from PTT/PET by conjugation method. Dual polymer (PTT/PET) heat shrinkage differences produce a permanent 3D crimp, which after heating brings in a permanent stretch. Recovery is $100 \%$, and it is anti-alkali, antichlorine, UV-resistant, dyeable and never- fatigue stretch. Even after a wash, the CM800 fabric has a wrinkle-free consistency that returns the material to its original smooth surface; there is 
no stretch fatigue even after repeated stretching. Its well-being includes dyeable dyestuff with dispense, which enables the fabric to maintain a pure color even after stretching. The shape-keeping is outstanding, as the CM800 spirals up after heat processing as a spring and returns after extending to its original peak. The wear resistance is good as that of filament yarn, which is not easily broken. Like that of filament yarn, which is not easily broken, the wear resistance is strong. Compared to spandex, and sorona, the CM800 is comfortable. The CM800's sustainable green source, guarantees that the fabric is skin friendly. More products, such as BI-CO Nylon, will be launched by the company [23].

\section{Primeflex stretch fabric}

PrimeflexTM is ideal for a broad range of uses, from sportswear to casual wear, business apparel, and fashion accessories due to its solid outstanding stretch, kickback, and lightweight properties [24]. The series versatile stretch fabrics like it were launched by Toray Industries, Inc. The Prime flex 2.0 is a variant of bicomponent nylon. The bicomponent structural nylon fiber bonds two nylon polymers with different performance characteristics into a single thread. The outcome is a hybrid yarn that can have stretch and soft feel features, both pretty vital components for the sports and athletic markets. This helps the fabric to adjust the contours of a body in motion, but also to return to its original form from stretching [25]. The Prime flex PET-recycled bico yarn structure of PTT (polytrimethylene terephithalate) derived partially from plant-based materials and recycled PET waste [26].

\section{Evolon ${ }^{\circledR}$}

Endless bi-component filaments are spun, deposited uniformly on a belt, then split into microfilaments simultaneously and entangled to produce Freudenberg's final Evolon ${ }^{\circledR}$ micro denier fabric using high pressure water jets. The method uses no solvents or chemical binders. Evolon $₫$ Microfilaments and Evolon® New Generation super-microfilaments are up to 200 times thinner than a human hair. Microfilament textiles from Evolon® can be customized to suit a range of needs and applications, including anti-mite bedding, cleaning cloths, sound absorption, technical packaging, printing media, sun protection, cosmetic wipes, and many other applications [27].

\section{Conclusion}

Bico fibers combine the benefits of both components, such as strength, hydrophilicity, low cost, etc. In the nonwoven industry, C/S bicomponent fibers are commonly used as bonding/binder fibers. Side-by- side and eccentric core-sheath bicomponent fibers are most commonly used to produce self-crimping yarns whereas a very complex extrusion called a segmented pie, or another called islands in the see is used to make micro denier fibers. Side-by-side and eccentric core-sheath bicomponent fibers are most widely used to develop self-crimping yarns, while micro denier fibers are generated by a very complex extrusion called a segmented pie or another called islands in the seas. In the coming years, the market for bi-component fiber is expected to increase considerably.

\section{Acknowledgement}

None.

\section{Conflict of Interest}

Authors declare no conflict of interest.

\section{References}

1. Mooney P, Shearer J, Mead J, Barry C, Truong Q, et al. (2018) Bicomponent Fiber Extraction Process for Textile Applications. Journal of Engineered Fibers and Fabrics 13(1)

2. Gopalakrishnan D (2019) Biocomponent fibres.

3. The Society of Fiber Science (2016) High-Performance and Specialty Fibers. (edn 1), p.451.

4. Purane SV, Panigrahi N (2007) Microfibres, microfilaments \& their applications. AUTEX Research Journal 7(3): 148-158.

5. (2018) \$2.26 Billion Bicomponent Fiber Market - Global Trend, Forecast, Competitive Analysis, and Growth Opportunity 2019-2024 ResearchAndMarkets.com

6. Naeimirad M, Zadhoush A, Kotek R, Neisiany RE, Khorasani SS, et al. (2018) Recent advances in core/shell bicomponent fibers and nanofibers: A review. Journal of Applied Polymer Science 135(21): 46265.

7. Liu Y (2004) Investigation of Fiber Splitting in Side-by-side Bicomponent Melt blown Nonwoven Webs through Post Treatment. University of Tennessee, USA.

8. Souissi M, Khiari R, Haddar W, Zaag M, Meksi N, et al. (2020) Dyeing of Innovative Bicomponent Filament Fabrics (PET/PTT) by Disperse Dyestuffs: Characterization and Optimization Process. Processes 8(5): 501.

9. Schütt G, Beyer S (2016) New system concepts for spinning bicomponent filament yarns. Asian Textile Journal: 28-31.

10. Plotz C (2020) Bicomponent fiber - get more than 2x. Manmade Fiber polymers.

11. Takematsu MM, Diniz MF, Mattos ED, Dutra RC (2018) Sheath-core bicomponent fiber characterization by FT-IR and other analytical methodologies. Polimeros-ciencia E Tecnologia 28(4): 339-347.

12. Kalabek NA, Babaarslan O (2016) Fiber Selection for the Production of Nonwovens. Non-Woven Fabrics.

13. (2010) Bicomponent Fiber.

14. Wilkie AE (1999) New Products from Bicomponents. West Melbourne, FL, International Nonwovens Journal, 8(1).

15. Hu J, Kumar B, Lu J (2020) Handbook of Fibrous Materials. Wiley-VCH Verlag GmbH \& Co. KGaA, pp. 973-1003.

16. Nakajima T, Advanced fiber spinning technology, spinning for nonwovens, ( $4^{\text {th }}$ edn), Woodhead Publishing Limited, Great Abington, UK.

17. Dayana Polyplast, Bio-Component Non-Woven.

18. Lu Z, Qian X (2010) Discussion on the Splitting Technique of Segmented Type Bicomponent Spunbonded Nonwovens, Proceedings of the 2010 International Conference on Information Technology and Scientific Management.

19. https://www.prnewswire.co.uk/

20. Sustainability Report 2020, Innovation for sustainable future.

21. https://connect.lycra.com/en/Fibers/T400

22. Creating Functionalities to the Bicomponent Fiber.

23. Castelino M (2017) Fibres \& Yarns expo: Getting innovative. 
24. Primeflex ${ }^{\mathrm{TM}}$, Bi-component Stretch Fabric.

25. (2020) Toray's Nylon Primeflex Now Available Worldwide.

26. (2021) The Textile Magazine.
27. Freudenberg, Evolon ${ }^{\circledR}:$ high-technology, outstanding properties, endless possibilities. 